\title{
Biomolecular imaging based on far-red fluorescent protein with a high two-photon excitation action cross section
}

\author{
Tsung-Han Tsai \\ Graduate Institute of Electro-Optical Engineering, National Taiwan University, Taipei 10617, Taiwan \\ Cheng-Yung Lin and Huai-Jen Tsai \\ Institute of Molecular and Cellular Biology and Angiogenesis Research Center, National Taiwan University, Taipei \\ 10617, Taiwan

\begin{abstract}
Szu-Yu Chen, Shih-Peng Tai, Kung-Hsuan Lin, and Chi-Kuang Sun
Department of Electrical Engineering and Graduate Institute of Electro-Optical Engineering, National Taiwan University, Taipei 10617, Taiwan
\end{abstract}

\begin{abstract}
The two-photon excitation action cross section of Hc-Red fluorescent proteins (Hc-RFPs) is measured and found to be of the same order as that of enhanced green fluorescent proteins. With a $618 \mathrm{~nm}$ emission wavelength in the far-red region and with an excitation wavelength around $1200 \mathrm{~nm}$, Hc-RPF-based two-photon fluorescence microscopy (2PFM) can offer deep penetration capability inside live samples and is ideal for in vivo gene expression study and biomolecular imaging in live objects. In vivo 2PFM of the developing heart deep inside a transgenic zebrafish embryo tagged by Hc-RFP is also successfully demonstrated. (C) 2006 Optical Society of America
\end{abstract} \\ Received October 14, 2005; revised January 7, 2006; accepted January 9, 2006; posted January 12, 2006 (Doc. ID 65391) \\ OCIS codes: $190.0190,110.0180,140.3580,260.2510$.
}

Transgenic lines carrying a tissue-specific green fluorescent protein (GFP) marker with emission wavelengths around $510 \mathrm{~nm}$ have been a powerful tool for the study of developmental biology because the spatial expression of GFP makes possible the encapsulation of the expression pattern of endogenous genes. ${ }^{1}$ Traditionally with a two-photon fluorescence microscopy ${ }^{2}$ (2PFM) based on a femtosecond Ti:sapphire (Ti:S) laser, green fluorescence can be excited by simultaneous absorption of two near-infrared (NIR) photons for high-resolution three-dimensional (3D) in vivo optical imaging. On the other hand, previous studies of human skin and other biological specimens have suggested that longer-wavelength light can penetrate live samples deeper, and the lowest light attenuation (due to the combined effects of absorption and scattering) in most biological materials exists around $1200-1350-\mathrm{nm} .^{3,4}$ It is thus highly desirable to develop long-wavelength fluorescentprotein-based 2PFM that would allow in vivo observations of gene expressions deep inside the developing embryos. This motivation is further strengthened by recent reports of multiphoton damage under excitation of femtosecond (fs) NIR light from a Ti:S laser. ${ }^{5,6}$ It was found that Chinese hamster ovary cells were unable to form clones with more than 2 $\mathrm{mW}$ average power under illumination by a 730-800 $\mathrm{nm}$ Ti:S fs laser. ${ }^{6}$ Recently the multiphoton photodamage induced by a NIR Ti:S fs laser has been widely used as a precise 3D nanosurgery tool. ${ }^{7,8}$ Tanabe and Takamatsu have shown that under fs $850 \mathrm{~nm}$ illumination, enhanced GFP (EGFP) can work as an effective phototoxic chromophore for inactivation of protein's function. ${ }^{9}$ On the other hand, our recent studies with a $1230 \mathrm{~nm}$ fs infrared (IR) source have shown much reduced multiphoton damage and prolonged viability in live biotissues. ${ }^{10,11}$ It is thus highly desirable to find an alternative fluorescence protein with an emission wavelength as long as possible that can be efficiently excited by fs light in the IR regions around $1200 \mathrm{~nm}$.

In this Letter, we report the measurement of the two-photon excitation action cross section of far-redemitting Hc-Red fluorescent proteins (Hc-RFPs), the value of which is found to be comparable to the maximal reported value of the highly efficient EGPF. The studied Hc-RFP was developed by a generated transgenic zebrafish line that has Hc-RFP expressed in myocardial cells. In vivo two-photon biomolecular three-dimensional imaging of the developing heart, tagged by the Hc-RFP deep inside the zebrafish embryos without removing the chorions, was also successfully demonstrated by using a $1230 \mathrm{~nm}$ fs light source, illustrating the high potential and capability of Hc-RFP-based 2PFM.

Hc-RFP is a fluorescent protein with an emission peak around $618 \mathrm{~nm}$ that was developed by random and site-directed mutagenesis of a nonfluorescent tetremeric chromoprotein from the reef coral Heteractis crispa. ${ }^{12}$ With a bright fluorescence and a relatively long emission wavelength compared with the commonly adopted Rs-Red, Hc-RFP can therefore be considered one of the most suitable choices for gene expression and protein localization studies and is selected as our studied live marker. We have successfully generated a new transgenic zebrafish line by screening from the embryos injected with DNA fragment containing a heart-specific regulatory element 


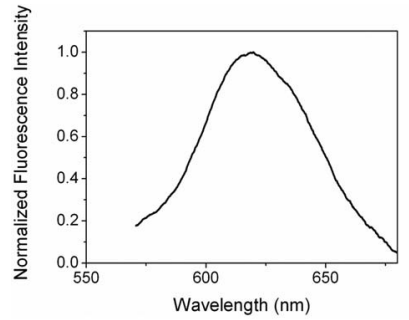

(a)

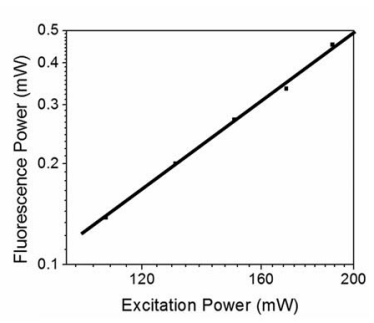

(b)
Fig. 1. (a) $2 \mathrm{PF}$ spectrum excited with a $1230 \mathrm{~nm}$ fs Cr:forsterite laser from Hc-RFP in myocardial cells of a live zebrafish embryo. (b) Dependence between $618 \mathrm{~nm}$ fluorescence power (squares) and $1230 \mathrm{~nm}$ excitation power measured from the Hc-RFP in myocardial cells of a live zebrafish embryo. The well-matched solid line is the slope $=2$ fit confirming the two-photon nature of light absorption. Note that both axes are log scaled.

of zebrafish cardiac myosin light chain 2 ( $\mathrm{cmlc2}$ ) gene fused with a Hc-RFP and an EGFP gene. ${ }^{13-15}$ Hc-RFP and EGFP were then expressed in the nuclei of cardiac cells for our two-photon fluorescence (2PF) studies. For the cross-section measurement, samples with the same Hc-RFP and EGFP concentrations were purified from transgenic zebrafish hearts co-tagged with Hc-RFP and EGFP. ${ }^{13-15}$

The 2PF spectrum of Hc-RFP was first measured in live transgenetic fishes using a $1230 \mathrm{~nm}$ fs Cr:forsterite laser, while the measurement of the $2 \mathrm{PF}$ cross section was performed with a tunable fs optical parametric oscillator on purified samples. For these two studies, fs pulses were focused by a $20 \times$ objective (LMPlan/IR 0.4, Olympus) into samples. The fluorescence signal was collected with the objective, discriminated from the excitation light by a filter, and detected by a CCD-based spectrometer. Figure 1 shows the measured $2 \mathrm{PF}$ spectrum of Hc-RFP with the peak emission wavelength located at $\sim 618 \mathrm{~nm}$, which matches the peak emission wavelength under single-photon excitation. ${ }^{12}$ We measured the relation between the collected fluorescence power and the $1230 \mathrm{~nm}$ excitation power. The measured fluorescence power was found to be in quadratic dependence on the excitation power over the whole emission wavelength range (570-680 nm), confirming its twophoton nature. An example is shown in Fig. 1(b). However, the $2 \mathrm{PF}$ also contains a wavelength shorter than $610 \mathrm{~nm}$, which is the theoretical 2PF limit excited with a $1230 \mathrm{~nm}$ laser source with a $20 \mathrm{~nm}$ bandwidth. It was measured and found that the power dependence of the emission below $600 \mathrm{~nm}$ was also quadratic. This study suggests that the fluorescence below $610 \mathrm{~nm}$ could be generated due to the phononassisted $2 \mathrm{PF}$ process.

The two-photon excitation action cross section $\left(\sigma_{\mathrm{TPE}}\right.$, defined as the product of the two-photon absorption cross section and the fluorescence quantum yield $^{16}$ ) of the Hc-RFP in the transgenic lines was also measured, and its dependence on excitation wavelength is shown in Fig. 2. Because the concentrations of the Hc-Red and the EGPF in the prepared samples were the same, we excited the two-photon fluorescence of the EGFP with the $900 \mathrm{~nm}$ NIR beam from a Ti:S laser (Mira900, Coherent) for calibration.
The two-photon fluorescence of the Hc-Red was excited with an IR beam with a wavelength from 1120 to $1230 \mathrm{~nm}$ from an optical parametric oscillator excited by the same Ti:S laser (thus with the same repetition rate). We used the measured EGFP fluorescence power and its reported $\sigma_{\mathrm{TPE}}$ value ${ }^{17}$ as a reference and considered the difference between the pulse width and the excitation volume to calibrate the Hc-RFP $\sigma_{\mathrm{TPE}, \lambda}$ value, where the subscript $\lambda$ indicates the excitation wavelength. Thus the obtained maximal value of the $\sigma_{\mathrm{TPE}}$ is $36 \mathrm{GM}$ (1 GM $=10^{-50} \mathrm{~cm}^{4} \mathrm{~s} /$ photon) at an excitation wavelength of $1160 \mathrm{~nm}$, while the maximal reported $\sigma_{\mathrm{TPE}}$ of EGFP is $41.2 \mathrm{GM}$ under $920 \mathrm{~nm}$ excitation, ${ }^{17}$ indicating that $\mathrm{Hc}-\mathrm{RFP}$ is a highly efficient $2 \mathrm{PF}$ protein. Due to the localized excitation property of the two-photon processes, high two-photon excitation efficiency, and the relatively higher penetration capability of the excitation IR light and the $618 \mathrm{~nm}$ emission light, Hc-RPFbased 2PFM should thus allow in vivo 3D observation deep inside live tissues with minimum invasiveness. Also, due to the fact that the tolerable excitation peak intensity in tissues increases significantly as the excitation wavelength moves from the NIR toward the $1200 \mathrm{~nm}$ wavelength region, ${ }^{5-11,18-20}$ Hc-RPF-based 2PFM should potentially provide better penetration capability than EGFP-based systems and can be an ideal replacement for the current EGPF-based 2PFM. ${ }^{9}$

Using a fs Cr:forsterite laser with a wavelength of $1230 \mathrm{~nm}$, ${ }^{1}$ we have successfully achieved in vivo 2PFM of the developed heart deep inside live transgenic zebrafish embryos tagged by Hc-RFP without removing their chorions. 2PFM is similar to a previously reported higher-harmonic generation microscope ${ }^{11}$ but with a backward collection geometry for the excited fluorescence. Figures 3(a)-3(c) show reconstructed $3 \mathrm{D}$ in vivo $2 \mathrm{PF}$ heart images based on Hc-RFP with three different view angles taken at the prim-16 stage, $\approx 30 \mathrm{~h}$ postfertilization. The whole cellular structure of the developing heart can be clearly identified through the $2 \mathrm{PF}$ of the HcRPF. Our demonstration indicates that Hc-RPFbased $2 \mathrm{PFM}$ with a high-resolution $3 \mathrm{D}$ imaging capability allows us to capture subtle developmental information on the in vivo heart on the cellular level occurring deep inside live embryos. With a $1230 \mathrm{~nm}$

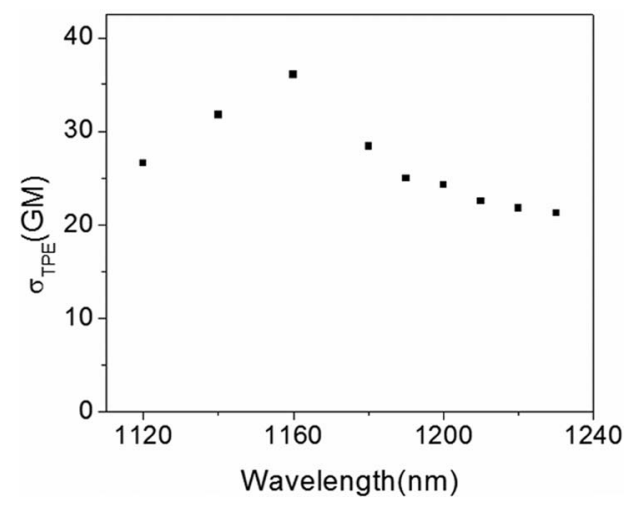

Fig. 2. Absolute two-photon excitation action cross sections $\left(\sigma_{\mathrm{TPE}}, y\right.$ axis $)$ of Hc-RFP. $1 \mathrm{GM}=10^{-50} \mathrm{~cm}^{4} \mathrm{~s} /$ photon. 


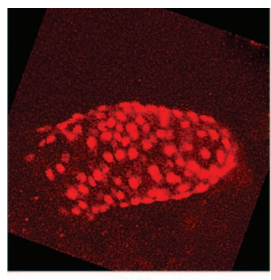

(a)

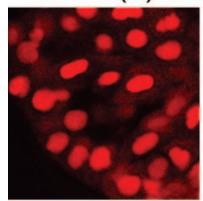

(d)

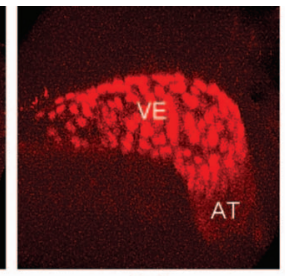

(b)

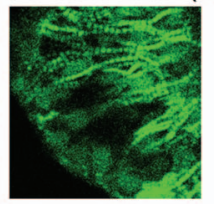

(e)

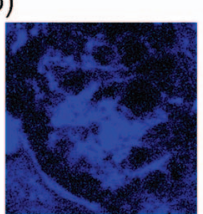

(f)

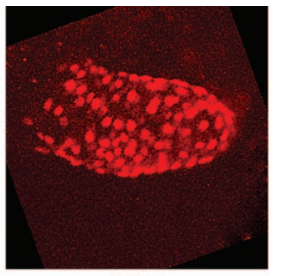

(c)

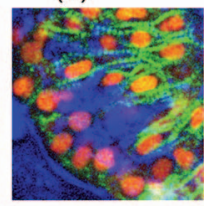

(g)
Fig. 3. (a)-(c) Reconstructed 3D in vivo two-photon fluorescence images of a live transgenic zebrafish heart taken at 30-hpf (prim-16 stage) without removing its chorion. (a) (c) Images with different view angles of $0^{\circ}, 60^{\circ}$, and $180^{\circ}$, respectively, rotating along the horizontal axis. AT, atrium, $\mathrm{VE}$, ventricle. Image size, $240 \mu \mathrm{m} \times 240 \mu \mathrm{m} \times 180 \mu \mathrm{m}$. (d) (g) High-resolution cross-sectional in vivo images of the heart surface of a live transgenic zebrafish taken at the prim-16 stage without removing its chorion: (d) $2 \mathrm{PF}$ image, (e) SHG image, (f) THG image, (g) combined image. Image size, $60 \mu \mathrm{m} \times 60 \mu \mathrm{m}$.

light, it is also advantageous to collect the forwardpropagating higher-harmonic generation signals ${ }^{11}$ for simultaneous multimodal imaging. ${ }^{21}$ Figures $3(\mathrm{~d})-3(\mathrm{~g})$ also show high-resolution cross-sectional in vivo images of the heart surface of the live transgenic zebrafish taken at the prim-16 stage without removing its chorion. While the $2 \mathrm{PF}$ image reflects the distribution of the nucleus of the myocardiac cells, second-harmonic generation provides the distribution image of the cardiac muscle fibers and thirdharmonic generation provides the image of myocardial cell membrane and the interfaces between the heart structure and its surrounding organs.

In summary, by successfully developing a zebrafish line tagged with Hc-RFP expression in the heart, the $2 \mathrm{PF}$ spectrum and the two-photon excitation action cross section of Hc-RFP were studied and measured. The two-photon excitation action cross section of HcRFP under IR light excitation was found to be comparable to the maximal reported value of EGFP. With a high two-photon emission efficiency and better penetration capability with the relatively longer emission and excitation wavelengths, Hc-RPF-based $2 \mathrm{PFM}$ thus provides a superb $3 \mathrm{D}$ in vivo deep tissue molecular imaging capability, offering deep insight into various genetic and molecular studies, including gene expression in vertebrate embryos. In vivo $2 \mathrm{PFM}$ imaging of the heart structure deep inside a developing transgenic zebrafish embryo tagged by Hc-RFP is also successfully demonstrated.

The authors gratefully acknowledge financial support from the National Health Research Institute of Taiwan (NHRI-EX94-9201EI) and the National Taiwan University Center for Genomic Medicine. C.-K. Sun's e-mail address is sun@cc.ee.ntu.edu.tw.

\section{References}

1. C.-J. Huang, C.-T. Tu, C.-D. Hsiao, F.-J. Hsieh, and H.-J. Tsai, Dev. Dyn. 228, 30 (2003).

2. W. Denk, J. H. Strickler, and W. W. Webb, Science 248, 73 (1990).

3. R. R. Anderson and J. A. Parrish, J. Invest. Dermatol. 77, 13 (1981).

4. S.-W. Chu, I-S. Chen, T.-M. Liu, C.-K. Sun, S.-P. Lee, B.-L. Lin, P.-C. Cheng, M.-X. Kuo, D.-J. Lin, and H.-L. Liu, J. Microsc. 208(3), 190 (2002).

5. P.-C. Cheng, B.-L. Lin, F.-J. Kao, M. Gu, X. Gan, M.-K. Huang, and Y.-S. Wang, Micron 32, 661 (2001).

6. K. König, P. T. C. So, W. W. Mantulin, and E. Gratton, Opt. Lett. 22, 135 (1997).

7. K. König, I. Riemann, and W. Fritzsche, Opt. Lett. 26, 819 (2001)

8. M. F. Yanik, H. Cinar, H. N. Cinar, A. D. Chisholm, Y. Jin, and A. Bem-Yakar, Nature 432, 822 (2004).

9. T. Tanabe and T. Takamatsu, paper presented at the Focus on Microscopy, Jena, Germany, March 20-23, 2005.

10. I.-H. Chen, S.-W. Chu, C.-K Sun, P.-C. Cheng, and B.-L. Lin, Opt. Quantum Electron. 34, 1251 (2002).

11. C.-K. Sun, S.-W. Chu, S.-Y. Chen, T.-H. Tsai, T.-M. Liu, C.-Y. Lin, and H.-J. Tsai, J. Struct. Biol. 147, 19 (2004).

12. N. G. Gurskaya, A. F. Fradkov, A. Terskikh, M. V. Matz, Y. A. Labas, V. I. Martynov, Y. G. Yanushevich, K. A. Lukyanov, and S. A. Lukyanov, FEBS Lett. 507, 16 (2001).

13. C.-J. Huang, C.-T. Tu, C.-D. Hsiao, F.-J. Hsieh, and H.-J. Tsai, Dev. Dyn. 228, 30 (2003).

14. A. J. Udvadia and E. Linney, Dev. Biol. 256, 1 (2003).

15. C.-D. Hsiao, F.-J. Hsieh, and H.-J. Tsai, Dev. Dyn. 220, 323 (2001).

16. C. Xu and W. W. Webb, J. Opt. Soc. Am. B 18, 481 (1996)

17. G. A. Blab, P. H. M. Lommerse, L. Cognet, G. S. Harms, and T. Schmidt, Chem. Phys. Lett. 350, 71 (2001).

18. U. K. Tirlapur, K. König, C. Peuckert, R. Krieg, and K.-J. Halbjuber, Exp. Cell Res. 263, 88 (2001).

19. P. E. Hockberger, T. A. Skimina, V. E. Centonze, C. Lavin, S. Chu, S. Dadras, J. K. Reddy, and J. G. White, Proc. Natl. Acad. Sci. U.S.A. 96, 6255 (1999).

20. J. M. Squirrell, D. L. Wokosin, J. G. White, and B. D. Bavister, Nat. Biotechnol. 17, 763 (1999).

21. S.-W. Chu, I.-H. Chen, T.-M. Liu, P. C. Cheng, C.-K. Sun, and B.-L. Lin, Opt. Lett. 26, 1909 (2001). 